\title{
The film antenna for capsular endoscope
}

\author{
Shigehiro Kai ${ }^{1 a)}$ and Masaharu Takahashi ${ }^{2 b)}$ \\ ${ }^{1}$ Graduate School of Engineering, Chiba University, \\ 1-33 Yayoicho, Inage-ku, Chiba-shi, Chiba 263-8522, Japan \\ ${ }^{2}$ Center for Frontier Medical Engineering, Chiba University, \\ 1-33 Yayoicho, Inage-ku, Chiba-shi, Chiba 263-8522, Japan \\ a)kaizirushi@chiba-u.jp \\ b) omei@faculty.chiba-u.jp
}

Abstract: Recently, the capsular endoscope that has extra function has been investigated. Although the power consumption tends to increase as the ability increases, it is difficult to increase the capacity of the battery due to the volumetric problem. In this study, we proposed an antenna for both wireless power transmission and image transmission to be mounted on a capsule endoscope. In this paper, we discussed the requirements desired to antennas to be mounted in capsular endoscope.

Keywords: capsular endoscope, wireless power transmission, FPC

Classification: Antennas and Propagation

\section{References}

[1] T. Kumagai, K. Saito, M. Takahashi, and K. Ito, "Design of receiving antenna for microwave power transmission to capsular endoscope," IEEE MTT-S International Microwave Workshop Series on Innovation Wireless, Uji, Kyoto, Japan, pp. 145-148, May 2011. DOI:10.1109/IMWS.2011.5877109

[2] S. Tsuzaki, K. Saito, M. Takahashi, and K. Ito, "Development of antenna for wireless power transmission to capsular endoscope," IEICE Commun. Express, vol. 3, no. 4, pp. 138-143, Apr. 2014. DOI:10.1587/comex.3.138

[3] S. Kai and M. Takahashi, "Investigation of the film antenna for wireless power transmission to the capsular endoscope," International Symposium on Antennas and Propagation, Okinawa, Japan, pp. 348-349, Oct. 2016.

[4] Q. Wang, K. Wolf, and D. Plettemeier, “An UWB capsule endoscope antenna design for biomedical communications," Applied Sciences in Biomedical and Communication Technologies, Rome, Italy, Nov. 2010. DOI:10.1109/ISABEL. 2010.5702832

[5] S. H. Lee, J. Lee, Y. J. Yoon, S. Park, C. Cheon, K. Kim, and S. Nam, “A wideband spiral antenna for ingestible capsule endoscope systems: Experimental results in a human phantom and a pig," IEEE Trans. Biomed. Eng., vol. 58, no. 6, pp. 1734-1741, June 2011. DOI:10.1109/TBME.2011.2112659

[6] S. Yun, K. Kim, and S. Nam, "Outer-wall loop antenna for ultrawideband capsule endoscope system," IEEE Antennas Wireless Propag. Lett., vol. 9, pp. 1135-1138, Nov. 2010. DOI:10.1109/LAWP.2010.2094996

[7] P. M. Izdebski, H. Rajagopalan, and Y. Rahmat-Samii, "Conformal ingestible capsule antenna: A novel chandelier meandered design," IEEE Trans. Antennas Propag., vol. 57, no. 4, pp. 900-909, Apr. 2009. DOI:10.1109/TAP.2009. 2014598 
[8] R. Puers, R. Carta, and J. Thoné, "Wireless power and data transmission strategies for next-generation capsule endoscopes," J. Micromech. Microeng., vol. 21, no. 5, p. 054008, Apr. 2011. DOI:10.1088/0960-1317/21/5/054008

[9] R. Carta, J. Thoné, and R. Puers, "A 3D ferrite coil receiver for wireless power supply of endoscopic capsules," Procedia Chem., vol. 1, no. 1, pp. 477-480, Sept. 2009. DOI:10.1016/j.proche.2009.07.119

[10] T. Sun, X. Xie, G. Li, Y. Gu, Y. Deng, and Z. Wang, "A two-hop wireless power transfer system with an efficiency-enhanced power receiver for motionfree capsule endoscopy inspection," IEEE Trans. Biomed. Eng., vol. 59, no. 11, pp. 3247-3254, Nov. 2012. DOI:10.1109/TBME.2012.2206809

\section{Introduction}

Recently, various studies of capsular endoscopes have been investigated [1, 2, 3, $4,5,6,7,8,9,10]$. Capsular endoscopes are kind of medical device that have about $20 \mathrm{~mm}$ length and $10 \mathrm{~mm}$ diameter. They are used instead of ordinary endoscopes, because they are able to examine small intestine that have not able to be examined by conventional endoscopes. Capsular endoscopes can be examined while reducing burdens on patients, but they have several problems due to being battery driven. First, batteries installed in capsular endoscope has limited capacity, thus sometimes examination is over with not being enough. Second, harmful matter contained in batteries may leak and hurt to patient. These problems can be solved by wireless power transmission to the capsular endoscope. If capsular endoscope operates by wireless transmission instead of cell batteries, we do not have to worry about improving the driving time of the capsule endoscope and no disaster due to leakage of harmful substances does not occur. Furthermore, it is not necessary to mount a battery in the capsule endoscope, so that the capsule endoscope can be miniaturized.

This paper presents a film antenna made by flexible printed circuit (FPC) for capsular endoscope. By using FPC, the risk of deformation of the antenna can be reduced. Moreover, it is easy to mass-produce it. The antenna works with microwave method wireless power transmission not resonant inductive coupling method nor inductive coupling method. By using microwave method, no coil is necessary against the resonant inductive coupling method or inductive coupling method. Furthermore, the antenna can be used by not only wireless power transmission but also captured images transmission. In the other words, it is unnecessary to mount two kinds of antennas in a capsule, and only one antenna should be mounted. The operating frequency is $433.92 \mathrm{MHz}$ in the ISM band.

\section{System}

Several antennas have already been proposed $[4,5,6,7]$ for transmission from inside the capsule, but few antennas are intended to receive power. Many of the antennas for power reception uses coils $[8,9,10]$, which is not suitable from the viewpoint of miniaturization. In this research, the antenna mounted in capsular endoscope can not only receive the power but also transmit pictures taken in intestine of patient. By radiating power from antennas installed on a body of patient 
and receiving by the antenna inside of capsule, capsular endoscope can take picture and transmit pictures taken in a body to outside of the body. By linking with two functions with one antenna, it is possible to be reduced space inside of capsular endoscope. Antennas installed in capsular endoscope should correspond various rotational state since capsular endoscope moves around the inside of body, in order to receive power stably. We designed an antenna with equal radiation characteristics around the axis.

\subsection{Internal antenna}

We analyzed the characteristic of the antenna put on a simplified human body model by using FDTD method. Power receiving antennas used in wireless power transmission currently using a coil have already been proposed and are mainstream, but antennas for transmitting images from inside of capsule to outside of body are separately required. In addition, although existing antennas for transmitting images are mainly deformed dipole antennas, in this research we adopt a loop antenna. The designed antenna is constructed by loop antenna and open stub structure. Normally, when we design loop antenna, its length should be one wave length, however it is impossible to install such a long element in a capsular endoscope, therefore by using open stub structure, we realized impedance matching. As a result, we succeeded in designing the antenna which is as small size as a thumb nail. By adopting the loop antenna as the basic structure, it becomes possible to receive regardless of the polarization plane of radio waves. In addition, since the shape is annular, radiation in the axial direction of the capsule can be suppressed compared to the dipole antennas, and it is assumed that strong radiation is obtained around the axis of the capsule. The antenna is assumed rolled in and inserted in capsular endoscope for radiating equally around axial rotation. This meets the condition that the antenna installed in capsular endoscope should adapting various rotation because capsular endoscope may be various rotational states.

The antenna model is shown in Fig. 1(a). This antenna is constructed by $0.4 \mathrm{~mm}$ thickness metal covered with FPC. Each dimension is shown in Fig. 1(a). The antenna is installed in a model of capsular endoscope shown in Fig. 1(b) constructed by Circuit Case (ABS resin: $\varepsilon_{r}=3.0, \sigma=0.0 \mathrm{~S} / \mathrm{m}$ ), Capsule Case (PTFE: $\varepsilon_{r}=2.0, \sigma=0.0 \mathrm{~S} / \mathrm{m}$ ), and Capsule Dome (acrylic resin: $\varepsilon_{r}=3.5$, $\sigma=0.0 \mathrm{~S} / \mathrm{m}$ ). Properties of antenna is shown in Fig. 1(c). The portion shown in red is a loop antenna, and the portion surrounded by blue are stub structure. Turn the antenna into a tubular shape and power the point where A and A' are connected.

\subsection{External antenna}

In order to convey information taken in intestine to outside of body, it is necessary to install another antenna on the body. In this study, we referred to and improved an antenna proposed by the past study [2]. The size of this antenna is adjusted for use in our research. This antenna is shown in Fig. 1(c). The antenna is constructed by $2 \mathrm{~mm}$ width metal on substrate $\left(\varepsilon_{r}=2.17, \sigma=0.0 \mathrm{~S} / \mathrm{m}\right)$. Since the antenna has very wide bandwidth, we adopted for transmission antenna in this study. In the other words, this antenna get less effect from human body than any other type of antenna because it is possible to corresponding to displacement of resonance 


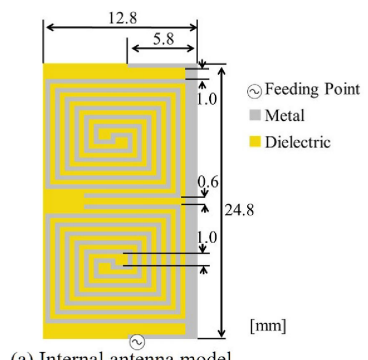

(a) Internal antenna model

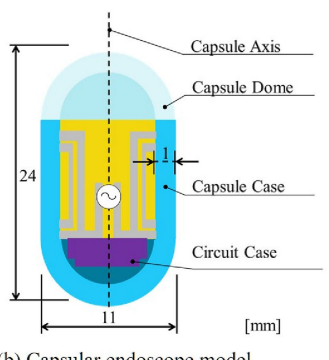

(b) Capsular endoscope model

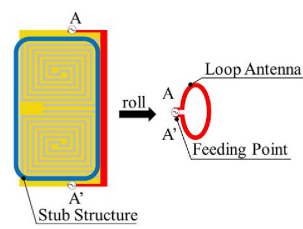

(c) Detail of antenna

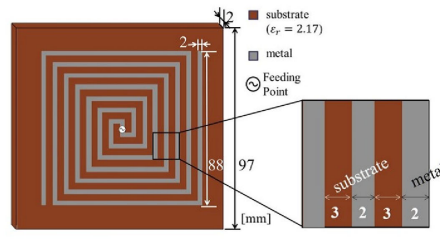

(d) External antenna model

Fig. 1. Antenna models
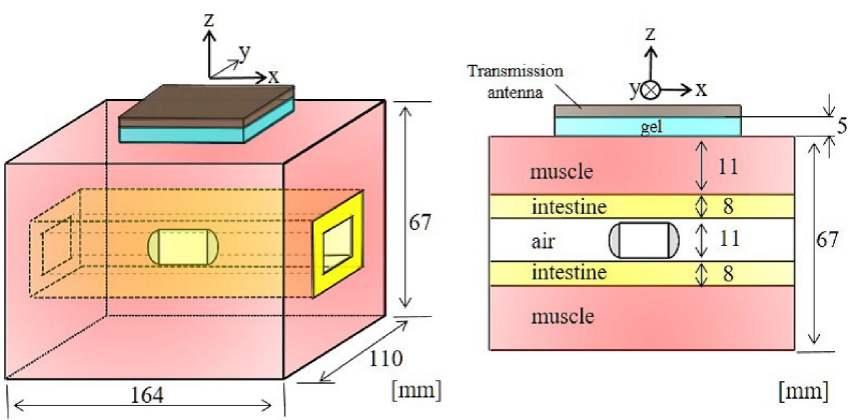

Fig. 2. Calculation model

frequency by dielectric constant. Furthermore, the antenna emits circular polarization wave, namely by using the antenna, it is possible to transmit power regardless of the rotational state of the capsular endoscope.

\subsection{Whole model}

In order to calculate the characteristics of the antenna, we used a simplified human body model instead of real human body. In this study, we used simplified twolayered model constructed by intestine and muscle.

Calculation model is shown in Fig. 2. The human body has simplified shape in order to shorten the calculation time. Calculation model is constructed by only muscle $\left(\varepsilon_{r}=57.7, \sigma=0.83 \mathrm{~S} / \mathrm{m}\right)$ and intestine $\left(\varepsilon_{r}=65.3, \sigma=1.92 \mathrm{~S} / \mathrm{m}\right)$. The external antenna is on gel $\left(\varepsilon_{r}=80.0, \sigma=0.0 \mathrm{~S} / \mathrm{m}\right)$ attached to the simplified human body model.

\section{Results}

Calculated and measured reflection coefficient of external antenna and proposed internal antenna are shown in Fig. 3(a). The external antenna maintains wideband characteristics even in the vicinity of the human body model in this research. The calculated reflection coefficient of the antenna was $-8.5 \mathrm{~dB}$. On the other hand, 


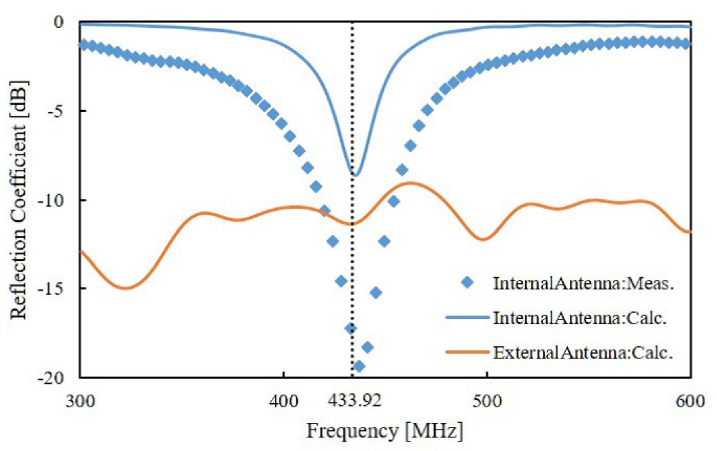

(a) Reflection coefficients

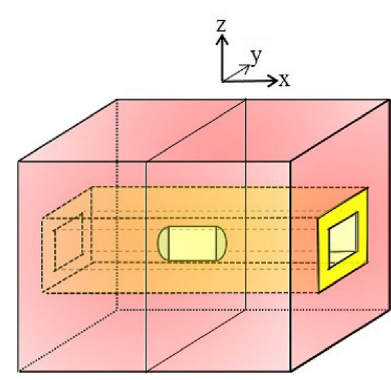

(b-1) Cut section

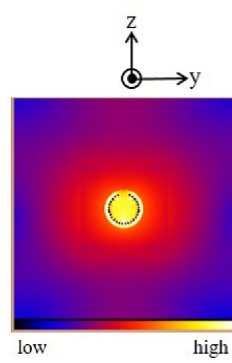

(b-2) Calculated E-field

Fig. 3. Results

measured reflection coefficient was $-17.2 \mathrm{~dB}$. Compared with the calculation results, baseline decreases depended on dielectric loss caused by FPC were observed in the actual measurement result, but the tendency was generally consistent. As a factor of errors between actual measurement and simulation, it is conceivable that errors due to electric constant of phantom, soldering and proximity of the coaxial cable and the phantom are considered, the greatest factor is the difference between the antenna model and the real antenna. In model, the film and the metal are arranged on the same plane and the same thickness due to constraints of calculation resource. However, in reality, metal thin films are arranged on the film, and these do not completely match. Since the actual antenna is strongly influenced by the dielectric loss tangent, the reflection coefficient of the measured value is deteriorated. The baseline drop in the measured value is about $-3 \mathrm{~dB}$, and even if this is taken into consideration, the actual antenna is not inferior to the simulation.

Calculated electric field intensity distribution is shown in Fig. 3(b). By the electric field intensity distribution, we observed that the antenna radiates equally around axial rotation of capsular endoscope. It satisfying the condition desired to antennas installed in capsular endoscope.

Calculated transmission efficiency from the external antenna to the internal antenna was $0.7 \%$. For practical use, it is required $2 \%$ of transmission efficiency. To solve this problem, we are staying addressed to improve external antenna and devise arrangement of the antenna. The external antenna used in wireless power transmission has no ground plate, thus it radiates both ways to simplified body and opposite. By attaching the ground plate to the antenna, the radiation direction of electric power is restricted. Thus leads to increasing transmission efficiency. 


\section{Conclusion}

We proposed an antenna that can be mounted in capsular endoscope. The antenna is expected not only transmit images taken in digestive organs but also receive power emitted by external of body. We observed that matching calculated reflection coefficient with measured one. As a result of calculation by FDTD method, the antenna radiates power equally round the axis. By wireless power transmission simulation, we acquired $0.7 \%$ transmission ratio and reflection coefficient at $433.92 \mathrm{MHz}$ was $-8.5 \mathrm{~dB}$. External antenna, it is necessary to properties to dissipate to one side. For practical use, it is required further improvement of the external antenna that is needed to dissipate to one side.

\section{Acknowledgments}

This work was supported by JSPS KAKENHI Grant Number JP26420338. 